\title{
A Refined Kinetic Analysis of Plasminogen Activation by Recombinant Bovine Tissue-Type Plasminogen Activator Indicates Two Interconvertible Activator Forms $^{\dagger}$
}

\author{
Laust B. Johnsen, ${ }^{\S}$ Peter Ravn,${ }^{\S, \perp}$ Lars Berglund, ${ }^{\S}$ Torben E. Petersen,*, ${ }^{*}$ Lone K. Rasmussen, ${ }^{\S}$ \\ Christian W. Heegaard, ${ }^{\S}$ Jan T. Rasmussen, ${ }^{\S}$ Connie Benfeldt," and Sergey N. Fedosov ${ }^{\S}$ \\ Protein Chemistry Laboratory, Department of Molecular and Structural Biology, University of Aarhus, Science Park, \\ Gustav Wieds vej 10 C, DK-8000 Aarhus C, Denmark, and MD Foods Research and Development Centre, R $\phi$ rdrumvej 2, \\ DK-8220 Brabrand, Denmark
}

Received March 23, 1998; Revised Manuscript Received June 18, 1998

\begin{abstract}
Bovine tissue-type plasminogen activator (tPA) was heterologously expressed in the methylotrophic yeast Pichia pastoris and characterized structurally and kinetically. The bovine single-chain tPAmediated activation of bovine plasminogen was studied in the presence and absence of fibrinogen fragments. We have proposed a refined new method of kinetic analysis which allows examination of both stationary and prestationary phases of this process. The investigation revealed the presence of two interconvertible forms of the recombinant bovine tPA being in equilibrium at a 1 to 50 ratio. Only the minor form was able to bind and activate plasminogen. Saturation of the whole pool of tPA required high plasminogen concentration $\left(K_{\mathrm{m}} \geq 5 \mu \mathrm{M}\right)$ in order to reverse the equilibrium between the two forms. Fibrinogen fragments activated the single-chain tPA due to preferential binding and stabilization of the minor "active" form of the enzyme until all the molecules of tPA were converted. The same mechanism could be applied to human tPA as well. The $K_{\mathrm{m}}$ values, obtained for recombinant bovine and human tPA in the presence of fibrinogen fragments, were found to be similar $\left(K_{\mathrm{m}}=0.1 \mu \mathrm{M}\right)$ while $k_{\text {cat }}$ of human tPA was 5-10 times higher.
\end{abstract}

The activation of plasminogen is an important process associated with degradation of an extracellular matrix such as dissolving of blood clots, tissue remodeling, and invasive growth of cancer cells. Tissue-type plasminogen activator $(\mathrm{tPA})^{1}$ and urokinase-type plasminogen activator (uPA) are the two major proteins responsible for conversion of plasminogen to plasmin. Although both tPA and uPA are related enzymes and activate plasminogen by cleavage of the same peptide bond, they have their own physiological features. The tPA-induced process is stimulated significantly on the surface of fibrin, and tPA is regarded as the fibrinolytic activator. On the other hand, the pericellular uPA-mediated activation of plasminogen is supposed to be engaged in tissue remodeling and cancer metastasis development.

The activation of plasminogen has been studied in detail in the human system from where the involved protein

This work is part of the FØTEK program supported by the Danish Dairy Research Foundation (Danish Dairy Board) and the Danish Government.

* Corresponding author.

$\S$ University of Aarhus.

"MD Foods Research and Development Centre.

${ }^{\perp}$ Present address: Biotechnological Institute, Kogle Allé 2, DK2970 Hørsholm, Denmark.

${ }^{1}$ Abbreviations: [Asp $\left.{ }^{1}\right]$ plasminogen, native form of bovine plasminogen with an aspartic acid at the $\mathrm{N}$-terminus; EACA, $\epsilon$-aminocaproic acid; $\mathrm{Fb}$, fibrinogen; $\left[\mathrm{Glu}^{1}\right]$ plasminogen, native form of human plasminogen with a glutamic acid at the N-terminus; PAI, plasminogen activator inhibitor; Pg, plasminogen; Pn, plasmin; tPA, tissue-type plasminogen activator; uPA, urokinase plasminogen activator. components have been identified and characterized. In contrast much less is known about plasminogen activation in other species. Bovine mastitis is an inflammatory disease of the mammary gland induced by various microorganisms, and a 20-fold increase in tPA activity has been reported in the milk of cows infected with Staphylococcus aureus (1). The activation of plasminogen by tPA is greatly increased by the $\alpha_{\mathrm{s} 2}$-casein dimer (2), and in order to study this system in more detail it is necessary to obtain bovine tPA. As this protein is only present in very small amounts in natural tissues and fluids and to our knowledge never has been purified, we have made recombinant expression of the protein.

tPA is a mosaic protein with five domains consisting of a finger domain, an epidermal growth factor domain, two kringle domains, and a serine proteinase domain. The finger and the second kringle are believed to be responsible for the interaction with fibrin $(3-5)$. tPA is synthesized as a single-chain polypeptide and can be converted into its more active two-chain form by plasmin scission of an Arg-Ile bond situated in the strand connecting kringle 2 with the serine proteinase domain (6).

Kinetic analysis of the human tPA catalysis was rendered in a number of papers (7-9) and resulted in several conclusions concerning its mechanism. The single-chain enzyme was considered as a poor catalyst when compared to the double-chain form. It was characterized by $K_{\mathrm{m}}=1-2$ $\mu \mathrm{M}$ toward $\left[\mathrm{Glu}^{1}\right]$ plasminogen and $k_{\mathrm{cat}}=0.3-0.6 \mathrm{~min}^{-1}(9)$. 
At the same time, the double-chain tPA had a considerably higher affinity to the substrate $K_{\mathrm{m}}=0.1-0.5 \mu \mathrm{M}$ as well as higher ability for plasminogen transformation $k_{\text {cat }}=4-5$ $\mathrm{min}^{-1}(8)$. All catalytic characteristics of the single-chain tPA improved when fibrin (or fibrinogen fragments) was added to the medium, showing the $K_{\mathrm{m}}$ and $k_{\text {cat }}$ values at the same level as those of the double-chain enzyme $(7,9)$. The double-chain tPA also demonstrated sensitivity to fibrin which was manifested in 5-10-fold increase of $k_{\text {cat }}$ but without any changes in $K_{\mathrm{m}}(8)$. The existence of an active ternary complex plasminogen-fibrin - tPA, converting bound plasminogen to plasmin, was proposed to be more favorable than the action of a fibrin-tPA complex toward free plasminogen (9). Previous publications about tPA kinetics mainly concerned the tPA-catalyzed reaction being in steady state. This is, in part, due to mathematical difficulties in the approximation of the complex reaction when the tPAcatalyzed conversion of plasminogen to plasmin was followed by cleavage of a measurable substrate by plasmin. The human tPA might be an inconvenient object for such investigations as well. Therefore, application of tPA from another source, provided with an adapted mathematical mechanism, could shed some light on "hidden" stages of the process. An appropriate candidate for this inquiry is bovine tPA, promising to be useful in several aspects.

We have expressed bovine tPA in the methylotrophic yeast Pichia pastoris and characterized the product from a structural and kinetical point of view. Comparison between human and bovine tPA required standardization of the experimental conditions, therefore activation of both enzymes was induced by bovine fibrinogen fragments. The bovine tPA was characterized by a slower equilibration with the components of the reaction medium when compared to human tPA. This allowed a detailed analysis of prestationary kinetics, impossible under the same conditions for the catalytic reaction performed with human tPA.

\section{MATERIALS AND METHODS}

Chemicals and Reagents. P. pastoris GS115 (his4) (10), the protease deficient strain SMD1168 (his4, pep4), and the expression vectors pPIC9K and pHIL-D2 were purchased from Invitrogen Corp. Super Taq polymerase was from HT Biotechnology, nucleotide triphosphates were from Pharmacia, Chameleon mutagenesis kit was from Stratagene, oligonucleotides were from DNA technology (Science park, Aarhus, Denmark), and all other enzymes were from New England Biolabs. PCR was performed in a Hybaid ABACUS thermal cycler. Sequencing was performed either with a Sequenase kit version 2.0 from United States Biochemical Corporation or with a dye terminator cycle sequencing kit from Perkin-Elmer. $\left[{ }^{35} \mathrm{~S}\right] \mathrm{dATP}$ was from Amersham International. Sequencing, ligation, transformation of Escherichia coli, DNA preparation, PCR, and other DNA modifying processes were performed according to the manufacturers' recommendations or standard laboratory procedures. Yeast media were composed as follows: YPD ( $1 \%$ yeast extract, $2 \%$ peptone, $2 \%$ dextrose), BMGY (1\% yeast extract, $2 \%$ peptone, $100 \mathrm{mM}$ potassium phosphate $\mathrm{pH} 6.0,1.34 \% \mathrm{YNB}$, $1 \%$ glycerol), BMMY (1\% yeast extract, $2 \%$ peptone, 100 $\mathrm{mM}$ potassium phosphate $\mathrm{pH} 6.0,1.34 \% \mathrm{YNB}, 1 \%$ methanol), MM (1.34\% YNB, $0.00004 \%$ biotin, $1 \%$ methanol), and $\mathrm{MD}(1.34 \% \mathrm{YNB}, 0.00004 \%$ biotin, $1 \%$ dextrose).

\section{KEX2 STE13}

\section{M..........EKR EAEA $\stackrel{\downarrow}{\downarrow}$ SYKVTC..... \\ $-89$ \\ $+1$}

FIGURE 1: Partial amino acid sequence of the pPIC9K/tPA secretion signal fused to the mature N-terminal of tPA is shown, as well as the expected two endoproteolytic split sites (KEX2, STE13).

Yeast extract, peptone, YNB, and casamino acids were from DIFCO, and G418 was from Life Technologies. Recombinant human tPA (Actilyse) and chromozym tPA $\left(\mathrm{CH}_{3}-\mathrm{SO}_{2}-\right.$ D-Phe-Gly-Arg-pNA) were from Boehringer Mannheim. S-2251 (H-D-Val-Leu-Lys-pNA) was from Chromogenix. Bovine [Asp ${ }^{1}$ plasminogen and PAI-1 were from American Diagnostica. PAI-2 was of the low molecular weight form and generously provided by Dr. I. Lecander (Lund, Sweden). $\mathrm{CNBr}$-fibrinogen fragments were made by incubation of 100 $\mathrm{mg}$ fibrinogen with $130 \mathrm{mg} \mathrm{CNBr}$ in $70 \%$ formic acid at room temperature for $16 \mathrm{~h}$. The resulting fibrinogen fragments were dialyzed against water for removal of low molecular weight fragments (membrane cut off $=12-14$ $\mathrm{kDa}$ ) and stored at $-80{ }^{\circ} \mathrm{C}$ at a concentration of $2.8 \mathrm{mg} /$ $\mathrm{mL}$. The bovine plasminogen used for zymography was purified as described in ref 11 , and plasminogen depleted bovine fibrinogen was obtained from Enzyme Research Laboratories.

Construction of the P. pastoris tPA Expression Vectors. pHIL-D2/tPA was derived from the $P$. pastoris integration vector $\mathrm{pHIL}-\mathrm{D} 2$. The tPA encoding region, including the native signal peptide, was inserted into the EcoRI restriction site in pHIL-D2, yielding pHIL-D2/tPA. Due to the presence of an internal EcoRI restriction site in the tPA cDNA, this was performed by PCR on pBtPA4 (12) with the forward 5'-ATGATGAGCGCAATGAAG-3' and reverse 5'-GGTGTCCCTGGTCATGG-3' primers. The EcoRI restriction site in the resulting amplified tPA encoding region was then methylated by EcoRI methylase and ligated to EcoRI linker oligonucleotides 5'-GGAATTCC-3'. Following digestion by EcoRI, the amplified fragment was ligated into pHIL-D2.

pPIC9K/tPA was derived from the $P$. pastoris integration vector PPIC9K. Using PCR, $5^{\prime}$ and $3^{\prime}$ NotI restriction sites were introduced into the bovine tPA cDNA using the plasmid pBtPA4 as template and the following forward and reverse primers, respectively, 5'-CTCAGGAGAGCGGCCGCATCGTAC- $3^{\prime}$ and 5'-GAGGAAAGCGGGCGGCCGCCCTGGG$3^{\prime}$. The resulting PCR product was cloned into the NotI restriction site in pPIC9K. To generate the native $\mathrm{N}$-terminus of tPA after cleavage by the signal peptidases (Figure 1), site specific mutagenesis was performed on pPIC9K/tPA, using the primer 5'-CTCGAGAAAAGAGAGGCTGAAGCTTCGTACAAAGTGACCTGCAGAGAT-3'. To ensure that the T4 polymerase replicated the entire plasmid, two nonmutagenic primers, situated in the Col E1 region (79617980) and HIS4 region (4801-4821) of pPIC9K/tPA, were included in the mutagenesis reaction. The tPA encoding region of pPIC9K/tPA was finally sequenced as a control for PCR-introduced mutations.

Transformation of P. pastoris with $p P I C 9 K / t P A$ and $p H I L-$ D2/tPA and Multicopy Colony Selection. P. pastoris strains GS115 (his4) and SMD1168 (his4, pep4) were transformed with the expression plasmids using the spheroblasting and 
electroporation techniques. $\mathrm{Mut}^{+}$and $\mathrm{Mut}^{\mathrm{s}}$ phenotypes were determined by screening for fast and slow growth on methanol-containing plates (MM plates) and, as a control, evaluating the growth of the same colonies on dextrosecontaining plates (MD plates). Multicopy colonies were selected by their ability to grow on YPD plates containing increasing concentrations of G418 $(0.25-4 \mathrm{mg} / \mathrm{mL})$. The transformation, phenotype determination, and selection were done essentially as described by the supplier.

Fermentation of $p P I C 9 K / t P A$ Transformed $P$. pastoris Strains. Cells were restreaked from freeze cultures on YPD or MD plates and a single colony was used for inoculation of $10 \mathrm{~mL}$ of BMGY medium. Cells were grown to log phase, and a suitable volume was used to inoculate $100 \mathrm{~mL}$ of BMGY medium, after which cells were grown until an $\mathrm{OD}_{600}$ of 2-6 was reached. Subsequently, the cells were centrifuged at $1000 \mathrm{~g}$ at room temperature, the BMGY medium was discarded, and the cells were resuspended into $200 \mathrm{~mL}$ of BMMY medium (with or without $1 \%$ casamino acids) in $2 \mathrm{~L}$ baffled shake flasks to an $\mathrm{OD}_{600}=1$. Cells were grown aerobically (shake flask covered with gaze) and supplemented with $1 \%$ methanol every $24 \mathrm{~h}$. Cells were removed by centrifugation, and the supernatant was stored at $-80{ }^{\circ} \mathrm{C}$. Cells were always grown in a rotary shaker at $30{ }^{\circ} \mathrm{C}, 300 \mathrm{rpm}$. When the expression levels of different strains were compared, fermentation was performed in a 12 $\mathrm{mL}$ reagent glass essentially as described above.

Purification of $t P A$. All steps were carried out at $4{ }^{\circ} \mathrm{C}$. Two hundred milliliters of the supernatant was thawed, centrifuged (10 min, $10000 \mathrm{~g}$ ), dialyzed against $20 \mathrm{mM} \mathrm{NaH}_{2}-$ $\mathrm{PO}_{4}, \mathrm{pH} 7.0,100 \mathrm{mM} \mathrm{NaCl}, 0.05 \%$ Tween 80, and applied to a column of $40 \mathrm{~mL}$ of lysine-Sepharose (prepared by reaction of $\mathrm{CNBr}-$ activated Sepharose $4 \mathrm{~B}$ with lysine). After the column was loaded, it was washed with $400 \mathrm{~mL}$ of 20 $\mathrm{mM} \mathrm{NaH} \mathrm{PO}_{4}, 200 \mathrm{mM} \mathrm{NaCl}$, and $0.05 \%$ Tween 80 and eluted with the same buffer containing $200 \mathrm{mM}$ L-arginine. Fractions were screened for tPA activity, pooled, and concentrated on an Amicon-30 membrane until $A_{280}$ was approximately 1 . The purified protein was stored at -80 ${ }^{\circ} \mathrm{C}$ for later use.

tPA Activity Assay and Zymography. tPA activity in international units (U) was determined by use of the chromogenic substrate chromozym t-PA according to the assay described by the manufacturer. The assay was performed in a total volume of $0.2 \mathrm{~mL}$ at $37^{\circ} \mathrm{C}$ in microtiter plates by the addition of the tPA sample to $0.1 \mathrm{M}$ Tris $\mathrm{pH}$ $8.5,0.15 \%$ Tween 80 , and $0.4 \mathrm{mM}$ chromozym t-PA. The reaction was followed at $405 \mathrm{~nm}$ over a period of $1 \mathrm{~h}$ in a thermostatically controlled Bio-Tek EL 340 BioKinetics Reader (Bio-Tek Instruments Inc., Winooski, VT). The amount of $\mathrm{U} / \mathrm{mL}$ was calculated as follows: (measured absorbance $\times \mathrm{min}^{-1} \times \mathrm{mL}^{-1}$ tPA sample $) \times(1 \mathrm{~cm} / 0.6 \mathrm{~cm}$, correction for light path in microtiter wells $) \times(1 / 9.75, \mathrm{U}$ conversion factor). The zymography was performed as described in ref 13 .

Coupled Peptidyl Anilide Plasminogen Activation Assay. The plasminogen activation potential of tPA was evaluated in a coupled peptidyl anilide assay, where the formation of plasmin was measured by its hydrolysis of the chromogenic substrate $\mathrm{S}-2251$. The plasminogen activation reaction was performed in a total of $0.2 \mathrm{~mL}$ containing $0.1 \mathrm{M}$ Tris $\mathrm{pH}$ 7.4, $0.02 \%$ Tween $80,0.03-1.08 \mu \mathrm{M}$ plasminogen, \pm 8.4 $\mu \mathrm{g}$ fibrinogen fragments, and $0.5 \mathrm{mM} \mathrm{S}-2251$. The reaction was initiated by addition of bovine tPA to the final concentration of $0.01 \mu \mathrm{M}$ in the absence of fibrinogen fragments or $0.001 \mu \mathrm{M}$ tPA in their presence. The concentration of human tPA was $3.3 \times 10^{-4} \mu \mathrm{M}$ in both cases. The reactions were carried out at $37{ }^{\circ} \mathrm{C}$ in microtiter plates and were followed at $405 \mathrm{~nm}$ over a period of $1 \mathrm{~h}$ in the same spectrometer as the tPA activity assay. For each assay, at least two independent experiments were made, with double determination in each experiment.

Mass Spectroscopy and Amino Acid Sequence Analysis. Automated Edman degradation was carried out on an ABI 477A/120A protein sequencer (Applied Biosystems) using standard programs. Mass spectra were acquired using a matrix-assisted laser desorption ionization (MALDI) mass spectrometer (Bruker BIFLEX, Bruker-Franzen, Bremen, Germany) equipped with a nitrogen ultraviolet laser at 337 $\mathrm{nm}$. Samples $(2 \mu \mathrm{L})$ dissolved in $0.1 \%$ trifluoroacetic acid were mixed with $2 \mu \mathrm{L} \alpha$-cyano-4-hydroxycinnamic acid (15 $\mathrm{g} / \mathrm{L})$.

Kinetic Analysis of the Coupled Peptidyl Anilide Plasminogen Activation Assay. Three kinetic models were designed in order to fit the experimental data. The basic model 1 implies the existence of two reactions: (i) transformation of plasminogen to plasmin catalyzed by tPA, and (ii) utilization of a chromogenic substrate by plasmin. A new method of linearization of the initial coordinates (product versus time) is proposed. A more complex scheme, described in model 2 , considers the existence of a prestationary step before the above reactions which distorts linearity of the plot in its initial part. Model 3 describes the behavior of the system in the presence of fibrinogen fragments.

Model 1. The dependence of tPA activity on the plasminogen concentration was investigated in the coupled reaction assay according to the following schemes

$$
\begin{gathered}
\mathrm{tPA}+\mathrm{Pg} \stackrel{K_{1}}{\Leftrightarrow} \mathrm{tPA}-\mathrm{Pg} \stackrel{k_{1}}{\longrightarrow} \mathrm{tPA}+\mathrm{Pn} \\
\mathrm{Pn}+\mathrm{S} \stackrel{K_{2}}{\Leftrightarrow} \mathrm{Pn}-\mathrm{S} \stackrel{k_{2}}{\longrightarrow} \mathrm{Pn}+\mathrm{P}
\end{gathered}
$$

where Pg represents plasminogen, Pn represents plasmin, $K_{1}$ and $K_{2}$ are the Michaelis constants $\left(K_{\mathrm{m}}\right)$ of the corresponding enzymes, and $k_{1}$ and $k_{2}$ are catalytic constants $\left(k_{\text {cat }}\right)$. The process was followed by conversion of the substrate S-2251 $(\mathrm{S})$ to the colored product $(\mathrm{P})$.

The usual analysis implies transformation of the coordinates $\left([\mathrm{P}]\right.$ versus time) and plotting of $[\mathrm{P}]$ versus $t^{2}(14)$. The curves after transformation are supposed to be linear in the initial part of the chart where concentrations of both $\mathrm{Pg}$ and $\mathrm{S}$ may be considered as constants and are equal to their initial values $[\mathrm{Pg}]_{0}$ and $[\mathrm{S}]_{0}$. The slopes of these lines are proportional to Michaelis equation for the first reaction which allows one to calculate $K_{1}$ and $k_{1}$ at known $K_{2}$ and $k_{2}$. This method has two major limitations: (i) the linear part can be manifested only at $[\mathrm{S}]_{0} \gg K_{2}$ and (ii) any prestationary kinetics would interfere with the accuracy of determination (like a lag phase or initial "jump"). Another model has been developed which includes correction for the decrease in $\mathrm{S}$ concentration (15).

We propose a simple kind of analysis which makes it possible to use any convenient concentration of $\mathrm{S}$ and fit 


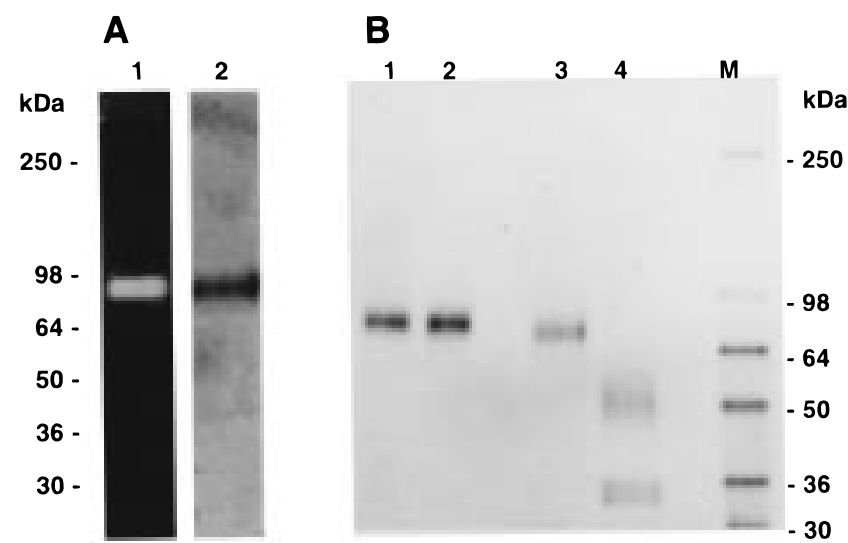

Figure 2: (A) Zymography (lane 1) and SDS-PAGE (lane 2) of one-chain tPA. (B) SDS-PAGE of one- and two-chain tPA. Lanes: (1) one-chain tPA unreduced, (2) two-chain tPA unreduced, (3) one-chain tPA reduced, (4) two-chain tPA reduced.

the curve in the whole range of $[\mathrm{P}]$. Reaction schemes S1a and $\mathrm{S} 1 \mathrm{~b}$ can be described by the corresponding system of differential equations:

$$
\begin{gathered}
\frac{\mathrm{d}[\mathrm{Pn}]}{\mathrm{d} t}=v_{\mathrm{a}}=\frac{k_{1}[\mathrm{PA}]_{0}[\mathrm{Pg}]}{K_{1}+[\mathrm{Pg}]} \\
\frac{\mathrm{d} p}{\mathrm{~d} t}=\frac{k_{2}[\mathrm{Pn}]\left(s_{0}-p\right)}{K_{2}+\left(s_{0}-p\right)}
\end{gathered}
$$

The tPA-reaction (S1a) under the chosen conditions was slow compared to the one catalyzed by $\mathrm{Pn}(\mathrm{S} 1 \mathrm{~b})$. It satisfied the requirement $[\mathrm{Pg}] \approx[\mathrm{Pg}]_{0}\left(v_{\mathrm{a}} \approx\right.$ const $)$ in the working time range and allowed a simple integration of eq 1a to [Pn] $=v_{\mathrm{a}} t$. After substitution of [Pn] in eq $1 \mathrm{~b}$ by $v_{\mathrm{a}} t$ and integration, the system was expressed as one equation:

$$
K_{2} \ln \left(s_{0} /\left(s_{0}-p\right)\right)+p={ }^{1} /{ }_{2} k_{2} v_{\mathrm{a}} t^{2}
$$

A simple transformation resulted in the linear dependence of $y$ on $t^{2}$

$$
y=y_{0}+v_{\mathrm{a}} t^{2}
$$

where $y=2 K_{2} / k_{2} \ln \left(s_{0} \mathrm{e}^{\left.\mathrm{p} / \mathrm{K}_{2} /\left(s_{0}-p\right)\right)}\right.$ and $y_{0}$ is the error in determination of the zero point $\left(y_{0} \approx 0\right)$.

The initial coordinates $p$ versus $t$ were transformed to $y$ versus $t^{2}$ using the known values of $K_{2}=250 \mu \mathrm{M}$ and $k_{2}=$ $1000 \mathrm{~min}^{-1}(16)$, as well as the value of $s_{0}$ from the experiment $(500 \mu \mathrm{M})$. The slope of an individual line was equal to $v_{\mathrm{a}}$ at the corresponding $[\mathrm{Pg}]$, and the experiments carried out at different $[\mathrm{Pg}]$ gave a set of lines with different slopes $\left(v_{\mathrm{a} 1}, v_{\mathrm{a} 2}, \ldots\right)$, see Figure 4 . The dependence of $v_{\mathrm{a}}$ on $[\mathrm{Pg}]$ can be fitted according to eq $1 \mathrm{a}$ in order to calculate parameters of the tPA-catalyzed reaction ( $K_{1}$ and $\left.k_{1}\right)$.

The presence of any prestationary kinetics would disturb the linearity of the chart $y$ versus $t^{2}$ in its initial part. It might be possible to evaluate the slope of the final linear component (reached at the stationary conditions) disregarding the initial shape of the curve if the prestationary step is relatively quick. One should be careful nominating the linear part of a curved dependence, as underestimation of the slope can be quite dramatic due to inappropriate choice.

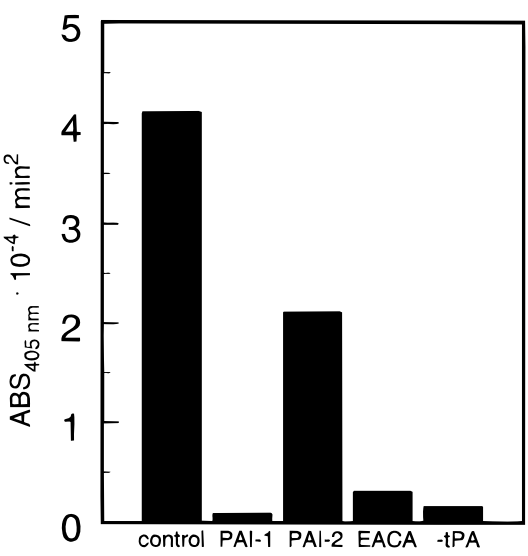

FIGURE 3: Inhibition of the bovine tPA-mediated plasminogen activation by PAI-1, PAI-2, and EACA in the presence of fibrinogen fragments. In the case of inhibition by PAI-1 and PAI-2, 2 pmol of tPA was incubated at room temperature for 20 min with 9 pmol PAI-1 or PAI-2 before addition to the reaction mixture consisting of $0.1 \mathrm{M}$ Tris $\mathrm{pH} 7.4,0.02 \%$ Tween $80,0.27 \mu \mathrm{M}$ Plg, $8.4 \mu \mathrm{g}$ fibrinogen fragments, and $0.5 \mathrm{mM} \mathrm{S}-2251$ in a total volume of 0.2 $\mathrm{mL}$. EACA was added to the reaction mixture just before initiation of the reaction. The reaction velocity was calculated as the slope of the line in a plot of the absorbance versus $t^{2}$.
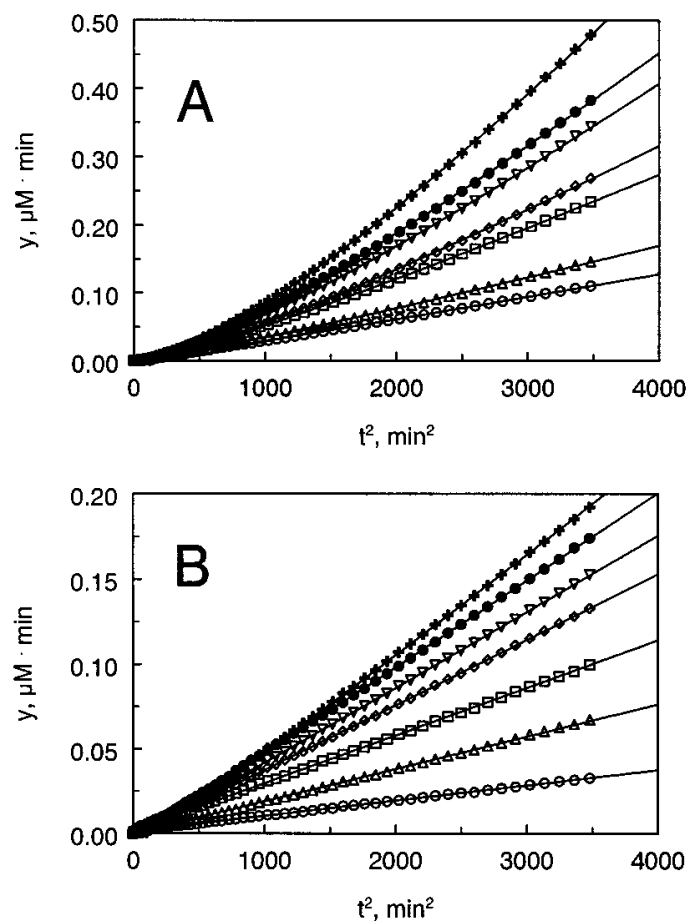

FIGURE 4: Release of $p$-nitroaniline after cleavage of the chromogenic plasmin substrate S-2251 in the reaction medium with tPA and $\mathrm{Pg}$ presented in the transformed coordinates $y$ versus $t^{2}$ (see eqs 3 and 6). Solid lines show the best fit according to eq 6 and model 2. (A) The dependence was obtained for bovine tPA. The symbols $\mathrm{O}, \triangle, \square, \diamond, \nabla, \bullet$, and + correspond to the Pg concentrations $0.084,0.19,0.30,0.41,0.52,0.63$, and $0.84 \mu \mathrm{M}$, respectively. (B) The dependence was obtained for human tPA. The symbols $\mathrm{O}, \triangle, \square, \diamond, \nabla, \boldsymbol{\bullet}$, and + correspond to the $\mathrm{Pg}$ concentrations $0.084,0.19,0.30,0.41,0.52,0.63$, and $0.73 \mu \mathrm{M}$, respectively.

Model 2. A more sophisticated approach allowed us to derive some information from prestationary kinetics as well. From Figure 4A one can see a well defined lag phase in the reaction with bovine tPA. Its expression increased proportionally to the added $\mathrm{Pg}$, and no linear component could be found at highest $[\mathrm{Pg}]$ in the used time scale. This observation 
pointed to a longer time of the prestationary reaction at high $[\mathrm{Pg}]$ when compared to that at low $[\mathrm{Pg}]$.

The simplest explanation of the observed phenomenon implied the existence of two interconvertible tPA conformations being in equilibrium according to reaction scheme $\mathrm{S} 2$

$$
\mathrm{tPA} * \underset{k_{-}}{\stackrel{k_{+}}{\rightleftarrows}} \mathrm{tPA}
$$

The form $\mathrm{tPA}^{*}$ cannot bind $\mathrm{Pg}$ and is referred to as the "inactive" enzyme while the "active" tPA is involved in the reaction with $\mathrm{Pg}$, see reaction schemes $\mathrm{S} 1 \mathrm{a}$ and $\mathrm{S} 1 \mathrm{~b}$. A lag phase in the dependence $y$ versus $t^{2}$ will be visible when the initial equilibrium $\mathrm{tPA}^{*} \rightleftarrows \mathrm{tPA}$ is shifted to $\mathrm{PPA}^{*}\left(k_{-} \gg k_{+}\right.$ , [tPA] $\left./\left[\mathrm{tPA}^{*}\right] \ll 1\right)$ and the conversion between these forms is relatively slow. The concentration of tPA at the beginning of the reaction was assumed to be approximately zero in order to minimize the number of parameters in the equations.

The appearance of Pn in time depends now not only on the velocity of the tPA reaction itself $\left(v_{\mathrm{a}}\right)$ but also on equilibration between $\mathrm{PA}^{*}$ and $\mathrm{tPA}$ :

$$
\begin{gathered}
\frac{\mathrm{d}[\mathrm{Pn}]}{\mathrm{d} t}=v_{\mathrm{a}}\left(1-\mathrm{e}^{-k * t}\right) \\
\text { where } v_{\mathrm{a}}=\frac{k_{1}[\mathrm{tPA}]_{0}[\mathrm{Pg}]}{K_{1}\left(1+\frac{k_{-}}{k_{+}}\right)+[\mathrm{Pg}]}, \quad k_{*}=k_{+}+\frac{k_{-}}{1+\frac{[\mathrm{Pg}]}{K_{1}}}
\end{gathered}
$$

The rate coefficient $k *$ is responsible for the expression of a lag phase (tPA* - tPA equilibration) and depends on the plasminogen concentration. Increase in $[\mathrm{Pg}]$ promotes a shift in favor of tPA + tPA-Pg which prolongs the equilibration and decreases the rate coefficient of the prestationary phase from $k_{*}=k_{+}+k_{-}$at $[\mathrm{Pg}] \rightarrow 0$ to $k_{*}=k_{+}$at $[\mathrm{Pg}] \rightarrow \infty$. The dependence of $k *$ on $[\mathrm{Pg}]$ has a Michaelis-like nature with the half saturation parameter equal to $K_{1}$ (tPA-Michaelis constant). Another parameter in eq $4\left(v_{a}\right)$ is a counterpart of $v_{\mathrm{a}}$ in eq 1a with the exception of the affinity to plasminogen reduced here by the factor $\left(1+k_{-} / k_{+}\right)$.

Integration of eq 4 gives a complex formula for [Pn] as a function of time:

$$
[\mathrm{Pn}]=v_{\mathrm{a}} t-\frac{v_{\mathrm{a}}}{k_{*}}+\frac{v_{\mathrm{a}}}{k_{*}} \mathrm{e}^{-k * t}
$$

Substitution of $[\mathrm{Pn}]$ in eq $1 \mathrm{~b}$ and integration provide the following expression of $y$ as a function of $t$ :

$$
y=y_{0}+v_{\mathrm{a}} t^{2}-\frac{2 v_{\mathrm{a}}}{k_{*}} t+\frac{2 v_{\mathrm{a}}}{k_{*}^{2}}\left(1-\mathrm{e}^{-k_{*} t}\right)
$$

where the notation for $y$ and $y_{0}$ is the same as before in eq 3 and $v_{a}, k_{*}$ are given in eq 4 . The chart $y$ versus $t^{2}$ has a tendency to be linear at the sufficiently long time of the reaction, i.e., $y \approx y_{0}+v_{\mathrm{a}} t^{2}$ at $t \gg 1 / k_{*}$. The curves from Figure 4 were fitted by a nonlinear regression program using eq 6 to calculate parameters $v_{\mathrm{a}}$ and $k_{*}$ at different $[\mathrm{Pg}]$. Their values were plotted against $[\mathrm{Pg}]$ and analyzed according to the corresponding formulas in eq 4 . The relevant constants for the tPA reaction $\left(k_{+}, k_{-}, k_{1}, K_{1}\right)$ were estimated.
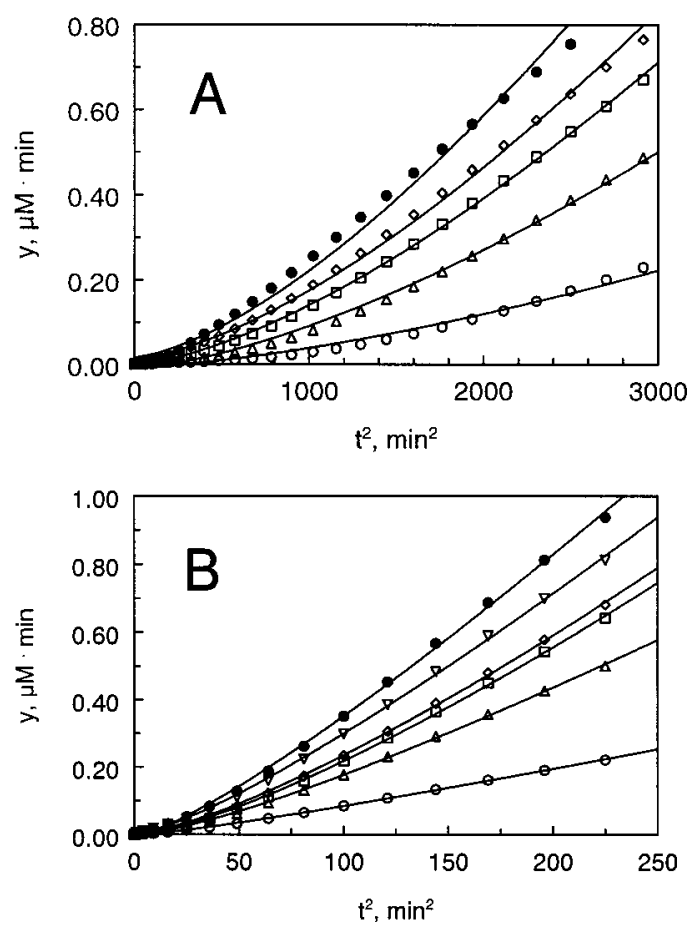

FIGURE 5: Release of $p$-nitroaniline after cleavage of the chromogenic plasmin substrate S-2251 in the reaction medium with tPA, $\mathrm{Pg}$, and fibrinogen fragments presented in the transformed coordinates $y$ versus $t^{2}$ (see eqs 3 and 6). Solid lines show the best fit according to eq 6 and the model 3. (A) The dependence was obtained for bovine tPA. The symbols $\bigcirc, \triangle, \square, \diamond$, and $\bullet$ correspond to the $\mathrm{Pg}$ concentrations $0.05,0.14,0.27,0.41$, and $0.68 \mu \mathrm{M}$, respectively. (B) The dependence was obtained for human tPA. The symbols $\bigcirc, \Delta, \square, \diamond, \nabla$, and $\bullet$ correspond to the $\mathrm{Pg}$ concentrations $0.05,0.14,0.27,0.41,0.54$, and $0.68 \mu \mathrm{M}$, respectively.

Model 3. The presence of fibrinogen fragments in the reaction medium somewhat complicated the analysis by adding another component to the system. The lag phase was already visible both for bovine tPA and human tPA (Figure $5 \mathrm{~A}, \mathrm{~B})$, and it was expressed at low $[\mathrm{Pg}]$ as well as at high [Pg]. Several models, analogous to reaction scheme S2, could have caused the appearance of the prestationary stage with one or another formula for $k_{*}$ in eq 6 . The scheme discussed below demands some limits for the value of $k$ * which makes it easy to accept or reject this model on the basis of appropriate or inappropriate fit.

$$
\mathrm{tPA}^{*} \underset{k_{-}}{\stackrel{k_{+}}{\rightleftarrows}} \mathrm{tPA}+\mathrm{Fb} \Longleftrightarrow \mathrm{Fb}-\mathrm{tPA}
$$

At high concentration of $\mathrm{Fb}$, reaction scheme $\mathrm{S} 3$ is reduced to the following description:

$$
\mathrm{tPA}^{*} \stackrel{k_{+}}{\longrightarrow} \mathrm{Fb}-\mathrm{tPA}
$$

The above model should be supplemented to reaction schemes S1a and S1b with Fb-tPA written instead of tPA.

The fitting can be performed on the basis of eq 6 , where $k_{*}=k_{+}$and the half-maximal value of $v_{\mathrm{a}}$ is reached at $[\mathrm{Pg}]$ $=K_{1}$. Our attempt to apply reaction scheme $\mathrm{S} 3 \mathrm{a}$ for human tPA (Figure 5B) was quite successful, see the Results. On the other hand, the fitting curves for bovine tPA (Figure 5A) showed lower accordance with the experimental data, which could imply existence of a more complex mechanism than 
the one discussed above. Nevertheless, the correlation coefficient of the generated curves was quite acceptable: $R^{2}$ $>0.99$.

The validity of calculations rendered on the basis of models 1,2 , and 3 and the corresponding equations was checked by a computer simulation. The theoretical curves were created by a program for modeling of a chemical reactions network using the nominated rate coefficients and the appropriate differential equations $(17,18)$. The results were reproduced by the program Gepasi available on the Internet (19) as well. The simulations showed the expected behavior of the system in response to the added reagents. Data of statistical analysis are presented as mean \pm standard deviation.

\section{RESULTS}

Optimization of Expression. The expression level of bovine tPA was optimized by a 3-fold strategy: (1) improving the secretion, (2) increasing the transcription of the tPA gene, and (3) minimizing the proteolytic breakdown of tPA in the expression media. First, expression levels were found to be $\sim 20$ times higher in pPIC9K/tPA recombinant strains, where secretion is driven by the signal peptide from the $\alpha$-mating factor, compared to expression levels from pHIL-D2/tPA strains, where secretion is driven by tPA's native signal peptide. Second, $\sim 10000$ colonies were screened on YPD plates containing different concentrations of G418 for multicopy colony selection. After incubation at $30^{\circ} \mathrm{C}$ for 4-5 days, colonies had appeared on all plates, with as few as two colonies on the YPD plates containing the highest G418 concentrations, demonstrating the stringency of the screening. The colonies scored from the plates with the highest concentration of G418, and thus presumably pPIC9K/ tPA multicopy strains, also exhibited the highest expression levels ( $\sim 2$-fold increase when compared to a single copy strain). Third, tPA activity was raised $\sim 3$-fold by inclusion of $1 \%$ casamino acids in the expression media. The use of the protease deficient strain (SMD1168, pep4) did not elevate the expression level further, compared to fermentation of GS115 strains with casamino acids included in the expression media. The various tPA expression levels during optimization were assessed by monitoring cell free supernatants by use of the tPA activity assay and found to reach maximum at $\sim 72 \mathrm{~h}$.

Purification and Structural Characterization. One pPIC9K/ tPA recombinant GS115 P. pastoris strain (no. 33, phenotype HIS4, G418 ${ }^{+}, \mathrm{Mut}^{+}$), scored from a YPD plate containing $4.0 \mathrm{mg} / \mathrm{mL} \mathrm{G} 418$ and showing the highest expression level of the tested colonies, was chosen for further fermentation. The yield of purified recombinant bovine tPA from $1 \mathrm{~L}$ of supernatant from this strain was $1.1 \mathrm{mg}$ of protein as determined by amino acid analysis or $0.63 \mathrm{U}$ as determined by the tPA activity assay.

SDS-PAGE of tPA, under reducing as well as unreducing conditions, showed a heterogeneous band at $80 \mathrm{kDa}$ (Figure 2 ), demonstrating that the purified tPA was present in its single-chain form. In the zymography a single lysis zone was seen at $80 \mathrm{kDa}$ (Figure 2). This molecular mass correlates with mass spectrometric data, where a broad mass peak was observed at $80 \mathrm{kDa}$ (results not shown). Three equally abundant $\mathrm{N}$-termini were seen by $\mathrm{N}$-terminal amino

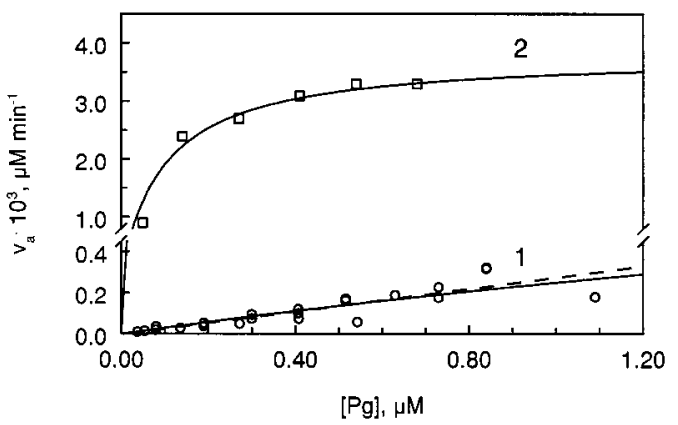

FIGURE 6: Dependence of the activity of bovine tPA $\left(v_{a}\right)$ on $[\mathrm{Pg}]$. (1) The reaction was performed with $1 \times 10^{-2} \mu \mathrm{M} \mathrm{tPA}$ in the absence of fibrinogen fragments. The solid curve $v_{a}=0.0015 \mathrm{Pg} /$ $(5+\mathrm{Pg})$ represents the best fit; see $v_{a}$ formula in eq 4 . The dashed line $v_{a}=0.00033 \mathrm{Pg}$ is shown as an example of alternative fit. (2) The reaction was performed with a decreased concentration of tPA $\left(1 \times 10^{-3} \mu \mathrm{M}\right)$ in the presence of fibrinogen fragments. The solid curve $v_{a}=0.0038 \mathrm{Pg} /(0.1+\mathrm{Pg})$ represents the best fit; see model 3.

acid sequence analysis of the recombinant tPA. One corresponded to the N-terminus of tPA, while the two others contained one or two copies of the Glu-Ala dipeptide situated amino terminal to the N-terminus of tPA (Figure 1).

Bovine tPA was converted from its single-chain form into its two-chain form by incubation with bovine plasmin, and sequencing of the three resulting chains separated by SDSPAGE (Figure 2) established that bovine tPA is cleaved by plasmin at the expected Arg278-Ile279 bond. The band at approximately $50 \mathrm{kDa}$ turned out to be the proteinase domain, even though it was $10-20 \mathrm{kDa}$ higher than the calculated mass $(29 \mathrm{kDa})$. The higher mass and blurry appearance of the band indicated a heavy and heterogeneous glycosylation of Asn451. N-terminal amino acid sequencing further showed that the two narrowly spaced bands at about $36 \mathrm{kDa}$ represented the same polypeptide, constituting the finger, EGF, kringle 1, and kringle 2 domains. The mass difference could indicate that the second potential glycosylation site (Asn117) was partially occupied. As expected from a yeast expression system, only $\mathrm{N}$-acetylglucosamine and mannose sugar residues could be detected when the produced tPA was subjected to carbohydrate analysis (performed by high $\mathrm{pH}$ anion exchange chromatography with electrochemical detection, data not shown).

The recombinant bovine tPA could be inhibited by the plasminogen activator inhibitors PAI-1 and PAI-2. As seen in Figure 3, the inhibition of 2 pmol of bovine tPA by PAI-1 was efficient, with complete inhibition of plasminogen activation by 9 pmol PAI-1. In contrast, bovine tPA was only $50 \%$ inhibited by addition of 9 pmol PAI-2 as judged from reduction of the plasminogen activation. As also seen in Figure 3, the addition of $10 \mathrm{mM} \epsilon$-aminocaproic acid (EACA), a lysine analogue, reduced the plasminogen activation velocity by $93 \%$.

Kinetic Characterization. Dependence of the tPA-activity $\left(v_{a}\right)$ and the prestationary rate coefficient $(k *)$ on plasminogen concentration were obtained from the charts $y$ versus $t^{2}$ (Figure 4,5 ) on the basis of models 1, 2, and 3 presented in the Methods section.

Bovine tPA showed a relatively low affinity to Pg in the absence of fibrinogen: $K_{\mathrm{m}} \approx 5 \mu \mathrm{M}, V_{\max } \approx 1.5 \times 10^{-3} \mu \mathrm{M}$ $\min ^{-1}\left(k_{\mathrm{cat}} \approx 0.15 \mathrm{~min}^{-1}\right)$, see Figure 6, curve 1, solid line. The $v_{a}$ chart was almost linear which could correspond 


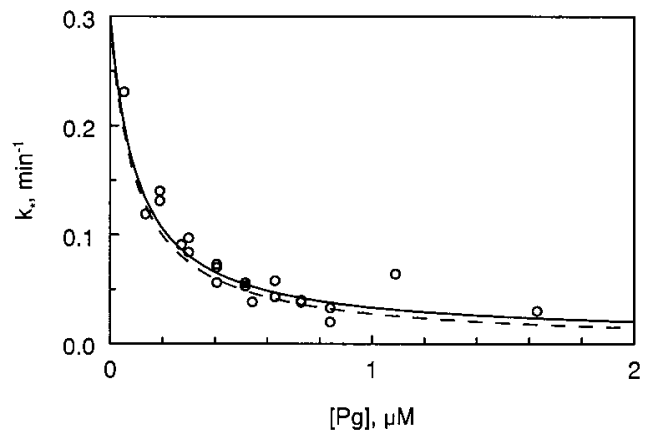

FIGURE 7: Dependence of the prestationary phase rate coefficient $(k *)$ on $[\mathrm{Pg}]$ obtained for bovine tPA without fibrinogen fragments. The chart was approximated according to the appropriate formula in eq 4. The solid curve represents the best fit $k *=0.3 \mathrm{Pg} /(1+$ $\mathrm{Pg} / 0.1)+0.006$ which corresponds to the ratio $k_{-} / k_{+}=50$; see model 2. The dashed curve $k_{*}=0.3 \mathrm{Pg} /(1+\mathrm{Pg} / 0.1)+0.0003$ is shown as an example of alternative fit which corresponds to the ratio $k_{-} / k_{+}=1000$; see model 2 .

to any value of the Michaelis constant if $K_{\mathrm{m}} \geq 5 \mu \mathrm{M}$ and $V_{\max } / K_{\mathrm{m}} \approx 0.3 \times 10^{-3} \mathrm{~min}^{-1}$, see Figure 6 , curve 1 , dashed line.

At the same time, analysis of the prestationary kinetics from the same experiment revealed the presence of a tPA form with high affinity to $\mathrm{Pg}\left(K_{\mathrm{m}} \approx 0.1 \mu \mathrm{M}\right.$, Figure 7) being in equilibrium with a low affinity form, see model 2 . The rate coefficient of the prestationary phase $k^{*}$ decreased from $k^{*}=k_{+}+k_{-} \approx 0.3 \mathrm{~min}^{-1}$ at $[\mathrm{Pg}] \rightarrow 0$ to $k^{*}=k_{+} \approx 0.006$ $\min ^{-1}$ at $[\mathrm{Pg}] \rightarrow \infty$ with the half-effect at $[\mathrm{Pg}] \approx 0.1 \mu \mathrm{M}$. The resulting model, consistent both with prestationary and stationary kinetics, can be written in the following form:

$$
\mathrm{tPA}^{*} \underset{0.3 \mathrm{~min}^{-1}}{\stackrel{\leq 0.006 \mathrm{~min}^{-1}}{\rightleftarrows}} \mathrm{PAA}+\mathrm{Pg} \stackrel{0.1 \mu \mathrm{M}}{\Longleftrightarrow} \mathrm{tPA}-\mathrm{Pg} \stackrel{\geq 0.15 \mathrm{~min}^{-1}}{\longrightarrow} \mathrm{Pn}
$$

The preparation of tPA under conditions of the assay consisted of a mixture of $\mathrm{PA}^{*}$ plus tPA, where the inactive form $\mathrm{PPA}^{*}$ was overabundant - the ratio $\left[\mathrm{tPA}^{*}\right] /[\mathrm{tPA}] \geq 50$. The suggested model makes a clear prediction that any compound, able to promote conversion $\mathrm{tPA}^{*} \rightarrow \mathrm{tPA}$, would increase the apparent affinity to Pg. The tPA reaction performed on the background of such a compound at $\mathrm{PPA}^{*}$ $\ll$ tPA would be characterized by the values of $K_{\mathrm{m}} \approx 0.1$ $\mu \mathrm{M}$. Fibrinogen fragments are candidates to cause the behavior of such a kind, and the model was proven by this test.

The presence of fibrinogen fragments in the system significantly activated bovine tPA, and the concentration of the enzyme was decreased 10-fold in order to simplify timedependent measurements. It should be mentioned that the steady state has not been achieved in the used time scale and $v_{a}$ values could not be nominated by linearization of the chart $y$ versus $t^{2}$ in Figure 5A. Analysis of the curves in Figure 5A according to model 3, see Methods, could be done equally well with different combinations of the parameters satisfying the following requirement: $k_{+} \leq 0.02 \mathrm{~min}^{-1}, K_{\mathrm{m}}$ $=0.1-0.3 \mu \mathrm{M}, k_{\text {cat }} \geq 1.5 \mathrm{~min}^{-1}$. The presented dependence of $v_{\mathrm{a}}$ on $\left[\mathrm{Pg}\right.$ ] (Figure 6, curve 2) with $K_{\mathrm{m}}=0.1 \mu \mathrm{M}$ and $V_{\max }=0.38 \times 10^{-3} \mu \mathrm{M} \mathrm{min}^{-1}\left(k_{\mathrm{cat}}=3.8 \mathrm{~min}^{-1}\right)$ had been obtained for the rate coefficient $k_{*}=k_{+}=0.005 \pm 0.001$ $\min ^{-1}$ assigned according to the $k_{+}$value calculated above.

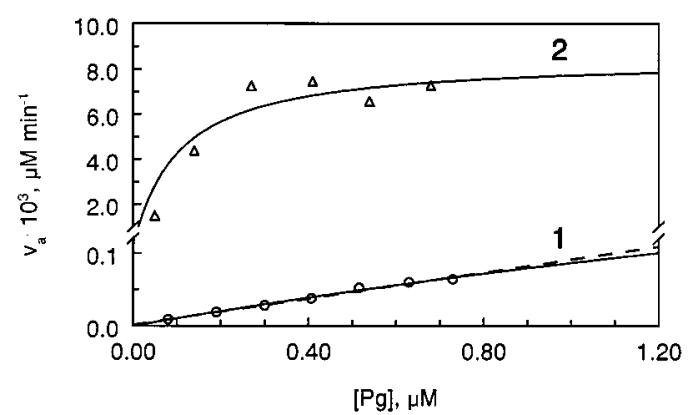

FIGURE 8: Dependence of the activity of human tPA $\left(v_{a}\right)$ on $[\mathrm{Pg}]$. (1) The reaction was performed with $3.3 \times 10^{-4} \mu \mathrm{M}$ tPA in the absence of fibrinogen fragments. The solid curve $v_{a}=0.0005 \mathrm{Pg} /$ $(4.5+\mathrm{Pg})$ represents the best fit; see model 2 . The dashed line $v_{a}$ $=0.0001 \mathrm{Pg}$ is shown as an example of an alternative fit. (2) The reaction was performed under the same conditions but in the presence of fibrinogen fragments. The solid curve $v_{a}=0.0085 \mathrm{Pg} /$ $(0.1+\mathrm{Pg})$ represents the best fit; see model 3 .

The reaction between human IPA and Pg was thoroughly assayed only under stationary condition according to the linear slopes of $y$ versus $t^{2}$ (Figure 4B) because the prestationary kinetics was too quick to allow any reliable calculations. Three curves with the highest $[\mathrm{Pg}]$ showed a systematic deviation from linearity in the beginning of the chart. Approximation by model 2 gave a rate coefficient of the prestationary phase decreasing to $k * \approx 0.2 \mathrm{~min}^{-1}$ at $[\mathrm{Pg}]$ $=0.73 \mu \mathrm{M}$. This value can be expected to be close to $k_{+}$ for human tPA. The stationary stage of the reaction revealed dependence of $v_{\mathrm{a}}$ on $[\mathrm{Pg}]$ with $K_{\mathrm{m}} \approx 4.5 \mu \mathrm{M}$ and $V_{\max } \approx 0.5$ $\times 10^{-3} \mu \mathrm{M} \mathrm{min}^{-1}\left(k_{\text {cat }} \approx 1.7 \mathrm{~min}^{-1}\right)$. It showed almost no advantage when compared to the linear fit $\left(v_{a}=1 \times\right.$ $10^{-4}[\mathrm{Pg}]$ ), Figure 8 , curve 1 , solid and dashed lines, respectively. It means that the reaction could be described by any values of $K_{\mathrm{m}}$ and $V_{\max }$ provided by $K_{\mathrm{m}} \geq 5 \mu \mathrm{M}$ and $V_{\max } / K_{\mathrm{m}} \approx 1 \times 10^{-4} \mathrm{~min}^{-1}$.

Fibrinogen fragments activated human tPA with the following change of the catalytic parameters: $K_{\mathrm{m}}=0.1 \mu \mathrm{M}$ and $V_{\max }=8.5 \times 10^{-3} \mu \mathrm{M} \mathrm{min}{ }^{-1}\left(k_{\text {cat }}=25 \mathrm{~min}^{-1}\right)$. At the same time, the prestationary phase became equally well expressed at all [Pg], see Figure 5B. The rate coefficient of this process revealed no $\mathrm{Pg}$ dependence and was equal to $k_{*}$ $=0.23 \pm 0.09 \mathrm{~min}^{-1}$. This value coincided with $k_{*}=k_{+}$ obtained at high $[\mathrm{Pg}]$ without fibrinogen fragments.

\section{DISCUSSION}

We have produced recombinant bovine tissue-type plasminogen activator in the yeast, $P$. pastoris. The initial expression levels were low from strains where secretion was driven by tPA's native signal peptide. However, expression levels were improved 100-fold by optimizing secretion, selecting for multicopy strains and minimizing proteolytic breakdown. After optimization of the expression, $0.63 \mathrm{U}$ or $1.1 \mathrm{mg}$ of tPA could be purified by affinity chromatography on lysine-Sepharose from $1 \mathrm{~L}$ of expression supernatant.

The molecular mass of the recombinant tPA was determined by SDS-PAGE, zymography, and mass spectroscopy to approximately $80 \mathrm{kDa}$. This molecular mass was considerably higher than the molecular mass of the naked tPA amino acid chain $(60 \mathrm{kDa})$ as well as that of natively glycosylated human tPA $(\sim 68 \mathrm{kDa})$. The produced tPA 


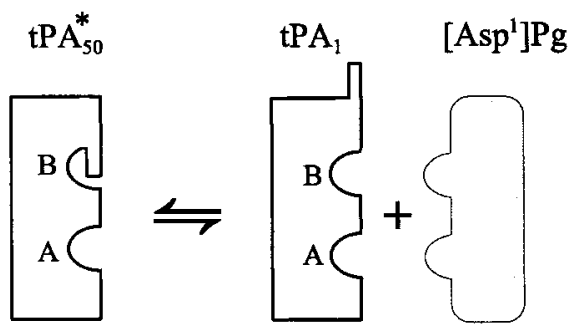

FIGURE 9: Two interconvertible forms of bovine tPA with high and low affinity for Pg. The form $\mathrm{tPA}^{*}$ possessing low affinity for $\mathrm{Pg}$ exists in equilibrium (50:1) with the high affinity form, tPA. It could be speculated that $\mathrm{PPA}^{*}$ only is restricted in its ability to bind Pg (binding interactions symbolized by the $\mathrm{B}$ site) while remaining catalytically active (active site symbolized by A).

therefore seemed to be heavily glycosylated. Bovine tPA contains two potential N-linked glycosylation sites, Asn117 and Asn 451. As judged from the band pattern of reduced two-chain tPA, a heterogeneous and heavy glycosylation of Asn451 appeared to be responsible for the increased mass of approximately $10-20 \mathrm{kDa}$ of the proteinase domain. The same band pattern also indicated a partial glycosylation of Asn117. The occurrence of two forms of tPA has been demonstrated in human tPA, where a partial glycosylation of Asn184 results in type I and type II tPA, which differ in their enzymatic turnover rates (20).

The kinetic analysis revealed the presence of two interconvertible forms of the recombinant bovine $\mathrm{tPA}^{*} \rightleftarrows \mathrm{tPA}$ where only tPA was able to bind Pg. The equilibrium between these forms in the preparation was shifted in favor of $\mathrm{tPA}^{*}(\geq 50: 1)$. It required high $[\mathrm{Pg}]$ to reach saturation of the enzyme by reversion of the equilibrium. Therefore, the apparent Michaelis constant was expected to be high, $K_{\mathrm{m}}^{\mathrm{app}}=K_{\mathrm{m}}\left(1+k_{-} / k_{+}\right) \geq 5 \mu \mathrm{M}$. In reality, affinity of the active form to Pg could be characterized by $K_{\mathrm{m}}=0.1 \mu \mathrm{M}$, according to the fitting of the prestationary lag phase, Figure 7. It has earlier been suggested that occupation of the lysyl binding site in kringle 2 by plasminogen would result in an "open" conformation of tPA (in contrast to a "closed" conformation when the lysyl binding site is occupied by an internal lysine residue) and that this "open" form would be more active against plasminogen (21). The "open" conformation could correspond to the active form of tPA in our model while the "closed" conformation matches the inactive form of tPA*. The possibility that the inactive and active forms of tPA reflect a gradual conversion of single-chain tPA into two-chain tPA during the assay can be neglected, since the rate constant for this process would have remained either constant or increased proportionally to $\mathrm{Pg}(\mathrm{Pn})$. This is definitely not the case for $k_{*}$ (Figure 7). One can speculate that the form tPA* is inactive solely due to its inability to bind Pg while it still possesses the potential catalytic activity (Figure 9).

Addition of fibrinogen fragments to the reaction medium was accompanied by a 50-fold increase in the affinity of bovine tPA to $\mathrm{Pg}$, giving the same $K_{\mathrm{m}}$ value as was determined in the prestationary phase without fibrinogen fragments. This fact points to involvement of fibrinogen in stabilization of the active tPA by incorporation of the enzyme in a multiprotein complex, presumably Fb-tPA and Fb-tPA$\mathrm{Pg}$. The plausible mechanism of this process is described above in model 3, and it implies preferential adsorption of the "active" tPA on fibrinogen: $\mathrm{tPA} * \mathrm{tPA}+\mathrm{Fb} \rightleftarrows \mathrm{FbtPA}$. The saturating concentration of $\mathrm{Fb}$ provides intensive shift to the form FbtPA with the rate-limiting step ascribed to the conversion PPA $^{*} \rightarrow$ tPA. This conversion was complete under the experimental conditions disregarding the concentration of added Pg. The produced complex FbtPA was then involved in the process of $\mathrm{Pg}$ activation and characterized by the same affinity to Pg as the free tPA. At the same time, it showed a $\approx 25$-fold increase of $k_{\text {cat }}$, when compared to the $\mathrm{Fb}$-free tPA.

In an attempt to compare the produced single-chain bovine tPA to commercial preparations of recombinant human single-chain tPA, equivalent kinetic studies were carried out for recombinant human tPA. The reaction kinetics with human tPA was similar to that obtained for the bovine enzyme, except for a very short prestationary phase, Figure 4B. If the model designed for bovine tPA could be directly applied to the human enzyme, the conversion $\mathrm{tPA}^{*} \rightleftarrows \mathrm{tPA}$ in the last case would occur relatively fast with $k_{*} \approx k_{+} \approx$ $0.2 \mathrm{~min}^{-1}$ at high $[\mathrm{Pg}]$. It made prestationary kinetics invisible at low $[\mathrm{Pg}]$ where $k_{*} \approx k_{+}+k_{-}$, though the ratio tPA*/tPA can be estimated as $\geq 45$ from the comparison of $K_{\mathrm{m}}$ values obtained with and without fibrinogen fragments. The presence of fibrinogen fragments together with human tPA caused some changes in characteristics of the lag phase, Figure 5B. It was seen at low $[\mathrm{Pg}]$ as well as at high $[\mathrm{Pg}]$ and its rate coefficient was Pg independent $\left(k_{*}=0.23 \mathrm{~min}^{-1}\right)$. This value was equal to $k_{*}$ for the conversion tPA* $\rightarrow$ tPA obtained for human tPA without fibrinogen fragments but on the background of high $[\mathrm{Pg}]$. The mechanism of activation of human tPA by fibrinogen fragments is presumably the same as for bovine tPA according to the values of calculated rate constants.

The values of $k_{\text {cat }}$ obtained for human TPA in the absence of fibrinogen fragments $\left(\geq 1.7 \mathrm{~min}^{-1}\right)$ and in their presence $\left(25 \mathrm{~min}^{-1}\right)$ were higher than those for bovine tPA under similar conditions. At the same time, the $K_{\mathrm{m}}$ values $(0.1$ $\mu \mathrm{M})$ were the same for both enzymes. This fact points to a quick association-dissociation between $\mathrm{PA}$ and $\mathrm{Pg}$, when compared to the rate of catalysis (i.e., the distinction in $k_{\text {cat }}$ does not influence $K_{\mathrm{m}}$ ). It is possible that the lower value of $k_{\text {cat }}$ observed for the bovine tPA is the result of a partial denaturation of the enzyme during fermentation and purification, giving a ballast protein in the bovine tPA preparation, but it could also simply be a consequence of native differences in the catalytic efficiency of bovine and human tPA toward bovine plasminogen. Alternatively, the heavy glycosylation of the proteinase domain could also influence $k_{\text {cat. }}$ Observations in favor of this latter possibility show that the nature of the oligosaccharide at Asn448 in the proteinase domain of human TPA influences both fibrin binding and clot lysis activity (22).

To characterize other properties of the produced tPA, inhibition of bovine single-chain tPA by PAI- 1 and PAI-2 was investigated. It was found that PAI-1 efficiently inhibited bovine single-chain tPA, while PAI-2 was a poor effector. These data are in agreement with observations for human tPA (23). In addition, EACA reduced the stimulation of the fibrinogen fragments upon the tPA-mediated plasminogen activation, confirming the interaction between bovine tPA and fibrin to be lysine dependent. 
In conclusion, we have produced recombinant bovine tPA. The preparation was tested by different methods including a physicochemical examination and a comprehensive kinetic analysis of the tPA-catalyzed conversion of plasminogen to plasmin. Advantages provided by the work with bovine tPA allowed us to characterize the behavior of the system under prestationary conditions of the enzymatic reaction both with and without fibrinogen. A new method of steady state reaction analysis applied to the tPA-mediated plasminogen activation was developed.

\section{ACKNOWLEDGMENT}

Special thanks to M. Vinther, A. Boisen, L. Møller, P. Mabhout, and R. E. Guldberg for skillful technical assistance, Dr. I. Lecander for the generous gift of PAI-2, and Dr. U. Christensen for a critical reading of the manuscript.

\section{REFERENCES}

1. Heegaard, C. W., Christensen, T., Rasmussen, M. D., Benfeldt, C., Jensen, N. E., Sejrsen, K., Petersen, T. E., and Andreasen, P. A. (1994) Fibrinolysis 8, 22-30.

2. Heegaard, C. W., Andreasen, P. A., Petersen, T. E., and Rasmussen, L. K. (1997) Fibrinolysis Proteolysis 11 (1), 2936.

3. Ichinose, A., Takio, K., and Fujikawa, K. (1986) J. Clin. Invest. $78,163-169$.

4. van Zonneveld, A., Veerman, H., and Pannekoek, H. (1986) Proc. Natl. Acad. Sci. U.S.A. 83, 4670-4674.

5. deMunk, G. A. W., Caspers, M. P. M., Chang, G. T. G., Pouwels, P. H., Enger-Valk, B. E., and Verheijen, J. H. (1989) Biochemistry 28, 7318-7325.

6. Ranby, M., Bergsdorf, N., and Nilsson, T. (1982) Thromb. Res. 27, 175-183.

7. Strandberg, L., and Madison, E. L. (1995) J. Biol. Chem. 270, 2344-23449.
8. Willmott, N., and Gaffney, P. (1995) Fibrinolysis 9, 347251.

9. Horrevoets, A. J. G., Pannekoek, H., and Nesheim, M. E. (1996) J. Biol. Chem. 272, 2183-2191.

10. Cregg, J. M., Barringer, K. J., Hessler, A. Y., and Madden, K. R. (1985) Mol. Cell. Biol. 5, 3376-3385.

11. Benfeldt, C., Larsen, L. B., Rasmussen J. T., Andreasen, P. A., and Petersen, T. E. (1995) Int. Dairy J. 5, 577-592.

12. Ravn, P., Berglund, L., and Petersen, T. E. (1995) Int. Dairy J. 5, 605-617.

13. Andreasen, P. A., Nielsen, L. S., Kristensen, P., GrøndahlHansen, J., Skriver, L., and Danø, K. (1986) J. Biol. Chem. 261, 7644-7651.

14. Chibber, B. A. K., Morris, J. P., and Castellino, F. J. (1985) Biochemistry 24, 3429-3434.

15. Christensen, U., and Müllertz, S. (1977) Biochem. Biophys. Acta 480, 275-281.

16. Wohl, R. C., Sinio, L., Summaria, L., and Robbins, K. C. (1983) Biochim. Biophys. Acta 745, 20-31.

17. Fedosov, S. N. (1994) Biochem. Biophys Acta. 1208, 238246.

18. Fedosov, S. N., Petersen, T. E., and Nexø, E. (1995) Biochemistry 34, 16082-16087.

19. Mendes, P. (1997) TIBS 22, 361-363.

20. Mori, K., Dwek, R. A., Downing, A. K., Opdenakker, G., and Ruud, P. M. (1995) J. Biol. Chem. 270, 3261-3267.

21. Bakker, H. F. A., Weening-Verhoeff, E. J. D., and Verheijen, J. H. (1995) J. Biol. Chem. 270, 12355-12360.

22. Wittwer, A. J., Howard, S. C., Carr, L. S., Harakas, N. K., Feder, J., Parekh, R. B., Rudd, P. M., Dwek, R. A., and Rademacher, T. W. (1989) Biochemistry 28, 7662-7669.

23. Lijnen, H. R., Bachmann, F., Collen, D., Ellis, V., Pannakoek, H., Rijkens, D. C., and Thorsen S. (1994) J. Int. Med. 236, 415-424.

BI9806697 